Proceedings

\title{
Use of Robots for Play by Children with Cerebral Palsy ${ }^{+}$
}

\author{
Xabier Gardeazabal $*, \$, \subseteq($ and Julio Abascal $\neq$ (1) \\ Egokituz Laboratory of HCI for Special Needs, Computer Architecture and Technology dep. at the University of \\ the Basque Country (UPV/EHU), Manuel Lardizabal Ibilbidea 1, 20018 Donostia, Gipuzkoa, Spain; \\ julio.abascal@ehu.eus \\ * Correspondence: xabier.gardeazabal@ehu.eus; Tel.: +34-943-015-113 \\ + Presented at the 13th International Conference on Ubiquitous Computing and Ambient Intelligence UCAmI \\ 2019, Toledo, Spain, 2-5 December 2019. \\ $\ddagger$ These authors contributed equally to this work.
}

Published: 21 November 2019

check for updates

\begin{abstract}
Free play is of great benefit for children's physical and cognitive development, especially for younger ages. However, children with motor restrictions cannot engage in free play like their peers because they face strong challenges to manipulate and interact with their environment. This lack of play opportunities may hinder proper cognitive development, along with several other problems such as decreased social skills or low self-esteem. In recent years several studies have discussed the use of robots to provide children with motor disabilities more opportunities for free play. This paper gives an overview of recent studies regarding the use of robots for play by children with Cerebral Palsy (CP). We also propose a work framework composed by a bi-manual articulated robot to further expand those children's play opportunities.
\end{abstract}

Keywords: free play; motor impairments; children with disabilities; Cerebral Palsy; assistive robots

\section{Introduction}

It has been widely established that free play has a key role in children's cognitive development. When children engage into free play, they tend to discover their capabilities, try out objects, make decisions, comprehend cause-and-effect relationships, learn, persist, and understand consequences of actions. Additionally, free play is spontaneous, intrinsically motivated, and self-regulated and requires the expressive personal involvement of the child [1]. Furthermore, play contributes to children's development in terms of discovery, learning, mastery, adaptation, creativity, self-expression and social skills [2].

There exists extensive literature supporting that motor movements in newborns play an important role for cognitive development at various levels (see [3] and the references therein). In summary, movements, motor schemes, and physical relationships with the real world can foster mental logic development, underpin logical construct, and are at the basis of the understanding of the cause and effect relationships and of the chaining of sequences of thoughts. Play provides exploratory means to children, through which they test their reality, and slowly build an elementary interpretation of the world by integrating perceptive and motor schemes together [3].

Section 2 gives an overview of the problems that children with limited motor function find when trying to engage into free play, while Section 3 gives a summary of some studies regarding the use of robots to promote free play among children with motor impairments. Section 4 describes the methodology of the study and our proposal for the use of a two-armed articulated robot to further help children with motor impairments, followed by final conclusions in Section 5 . 


\section{Effect of Physical disabilities on Free Play}

Some children lack self-initiated and spontaneous play activities, or have no other choice than to wait passively until someone interacts with them. As a consequence, these children may develop a learned helplessness can perpetuate the belief that they are unable to perform a task even though they may be able to $[4,5]$. Together with that feeling, they may also not perceive themselves as the owners of their own thoughts [3].

Additionally, the absence of play may affect children's perception of having control over the environment, and therefore they may end loosing motivation [6]. In fact, children with severe motor disabilities may regard themselves as spectators rather than actors, because they are used to "being done to" rather than "doing with" by their playmates or by adults in care of them, who tend to take the initiative and direction of the play [6]. The absence of play may also deprive the child from key interactions with the social and material environment, resulting in isolation and reduced social, linguistic and cognitive skills' development [2].

Children with motor disabilities may not have the same opportunities to engage in playful activities as their typically developing counterparts. The limitations for free and autonomous participation in daily activities due to motor impairments may hinder the proper development of children's sensory and perception capabilities, and cause low self-esteem and self-efficacy [3]. This is the case of children with Cerebral Palsy (CP), who have diminished play ability due to them having greater motor limitations [7].

$\mathrm{CP}$ is a physical impairment related to a problem of the central nervous system which creates substantial limitations to physical ability or motor skills, and may also create intellectual and neuro-psychological impairments, language and speech disorders, sensory disabilities, and emotional or social difficulties (Tingle 1990, as cited in [3]). The prevalence estimates of CP are generally decreasing globally since the last decade for the more severe subgroups, but there are still around $2 \mathrm{CP}$ cases per 1000 live births in developed and in developing countries [8].

In comparison to typically developing children, children with $\mathrm{CP}$ are generally less playful than those without CP [4], and are more compliant, less responsive, and less independent [9]. Additionally, children with less cognitive-behavioral problems in daily activities and/or higher gross motor function are more self-determined than those with more severe impairments [10]. Self-determination is described as taking responsibility for oneself and advocating for personal life goals [10], and it includes behaviors oriented towards meeting personal life goals such as identifying desires, actively pursuing interests, making decisions, and solving problems [4]. Therefore, improving the playfulness of children with CP might also improve their self-determination [4].

\section{Robot Mediated Play}

The use of robots as mediators for play has been shown to be beneficial in multiple levels for children with motor impairments. Schulmeister et al. [11] tested the applicability of small mobile and inexpensive robots for children's play and learning through a case study where one child learned to control two Lego Mindstorm robots through one and two switches mounted on the sides of their head. The results were very positive. As noted by the child's teacher, the child showed increased memory retention; learning skills; initiative during play; independence while playing; willingness to practice with robots during the study; and became more vocal. The teacher did not note any increase in the child's social skills except increased interest towards the researchers.

Schulmeister et al. also noted that the use of such robots for play are potentially beneficial for language development:

"The participant's almost continual positive affect and gaze shifting between the task and the experimenters were displays of positive joint attention. Joint attention is a precursor to language development. The participant's continual display of excited joint attention leads to the conclusion that this type of robot play would facilitate language development. " 
Robots have also been shown to increase playfulness. Rios-Rincon et al. [4] tested the effect on playfulness of a Lego robot being used by four children (5-9 years old) with motor disabilities (levels IV-V according to the GMFCS [12] \& MACS [13]). The experiments consisted of externally observing 4 mothers playing with their children. The children could remotely control the robot using Jelly Bean switches, which were appropriately placed for each child. Results showed that all children's playfulness levels increased significantly when they played with the robot. Furthermore, the results indicated that three children had retention of the improved playfulness level.

Despite their advantages, the authors pointed some drawbacks of Lego Invention systems, such as their lack of guaranteed accuracy in all of their movements [4]. They noted that some children were momentarily disappointed when the robot did not go in the exact expected direction, or even felt frustrated when commands of the controller did not reach the robot due to problems with an infrared signal. The study concluded that further play activities and strategies that will allow children with motor restrictions to express their playfulness need to be found.

In another study, Rios-Rincón et al. observed the changes in communicative utterances of a 7 year old child with CP and speaking limitations (only said "yes" and "no") while she played with her mother and with or without a Lego robot [14]. During the tests, the child's Yes/No utterances were tracked. Additionally, they coded the mother's utterances and they also tracked the number of times the mother performed a function on behalf of the child. Results showed that the child's utterances increased with the robot, which implies that the child became more communicative while using the robot.

Contrary to the authors' initial belief, the Direction of Action was higher when using the robot than without it. They reasoned that the mother may have tried to instruct the kid on how to operate the robot, because the child had only one training session about the use of the switches [14]. The authors noted that in order to give children the opportunity to develop adequate skills to operate the robot, extended training periods are required. Direction of Attention decreased with the use of the robot, showing that the mother's attempts to direct the child's behavior decreased as the child's independence during play increased. It was also noted that while using the robot, the mother went from directing what she wanted the child to do during play, to instead describe what the child was doing. Open-Ended Questions decreased with the robot, while Yes/No questions increased. The latter became increasingly varied and tended to regard the child's actions with the robot. Additionally, the mother spoke less on behalf of the child with the robot. According to Rios-Rincón et al., all this shows that the interaction became more reciprocal when using the robot, and an increase in the child's self or shared expression was measured [14].

\subsection{Robot Use to Evaluate Cognition}

Many standard tests used to measure developmental levels of children do not take into account that some of them may be affected by disabilities, and be faced with physical or further barriers when being tested through them. As a consequence, it is difficult to measure the developmental levels of children with disabilities, who may unfairly under-perform in the tests, giving the impression that they are less cognitively developed than they really are [15].

Some studies have focused on the opportunities that robots offer to evaluate the cognitive development of children with disabilities. Polletz et al. tested the applicability of a Lego Mindstorm robot to measure children's cognitive development by having 18 typically developing children aged 3-5 perform three tasks with the robot [15]. They specifically measured the ages at which typically developing children demonstrate understanding of means end causality, negation, binary relations and sequencing while using switches to control Lego Robots. The first task measured understanding of causality, by requiring the child to press and hold a switch until the roverbot knocked over a stack of blocks. The second tasks measured understanding of negation, by having the child help the researcher build a stack of blocks. The children were required to stop the roverbot beside a pile of blocks by releasing the same switch as before at the right time, so that the researcher could load the blocks onto 
the roverbot. Then, they were required to again stop the roverbot at the original stacked blocks location so that the researcher could unload the blocks. The third task measured understanding of binary logic and sequencing of two actions. The children were required to choose between two stacks of blocks, and then have the roverbot (placed between them, and facing away from the child) knock the chosen stack by using the appropriate one of two additional switches to make the roverbot turn left of right (binary logic) towards the chosen stack, and then using the original forward switch to knock the blocks (sequencing of actions).

The cognitive skills they identified (means end causality, negation, binary relations and sequencing) by performing low motor and linguistic demanding tasks with robots have, according to the authors, direct applicability to assistive technology use in broad scale. Therefore, it is important that these skills get meaningful attention [15].

\subsection{Virtual Play and Simulations of Manipulation}

Although it has not been specified whether motor movements are indispensable or not for promoting cognitive development, some authors claim that what is important is the intention of movement, and not movement itself, and therefore suggest that virtual simulations in which the user is given the impression that they are interacting with the environment is equivalent to actually doing it [3]. Perhaps in this regard, some studies have also tested the opportunities of simulated virtual robots and environments that mimic the previously tested physical robots (e.g., [16-19]). However, no definite conclusions pointing towards the prevalence of one over the other have been reached so far.

In one study, both a virtual and a physical IAMCAT (integrated augmentative manipulation and communication assistive technology) was tested in a school with nine children with disabilities and nine typically developing children [19]. The physical version of the IAMCAT was composed of Lego Mindstorms, while the virtual version was a simulation of the former. Encarnação et al. concluded that both systems proved to be useful to allow children with neuromotor disabilities to jointly participate with their peers and teachers in the academic activities within the classroom.

The teachers involved in that study adapted their curricular activities so that all children could perform them, regardless of the use of the IAMCATs, and believed that both systems helped towards the inclusion of children with disabilities regarding participation in the classroom and access to the academic curriculum [19]. The teachers also stated that the use of IAMCATs was generally positive both for the children in need of them as well as for the rest of the educational community. However, they also noted the added difficulties of managing such a class or the extra time required by children with disabilities to complete the activities, and they also mentioned the need for an additional educator within the classroom to better support the children using the IAMCAT system(s).

Among the study's limitations, the authors mention that despite using both a virtual and a physical system, this study did not draw any conclusions as to which version of the IAMCATs (virtual or physical) was better, as they did not perform any tests in that regard. Additionally, neither the children's experience while using the systems, nor their academic advances were tracked. It was only the teachers' opinions on the systems that were collected [19].

\section{Methodology}

Taking into account the studies mentioned in the previous sections, our thesis is that young children with severe motor function limitations encounter serious barriers or total inability to engage on true free play, and that high-precision robotic manipulators can not only improve the playfulness of such children, but that they can also help breach the gap towards free play opportunities. Additionally, we hypothesize that real-life play interactions are more effective than virtually simulated ones for stimulating young children's cognitive development. Therefore, the purpose of this study is to develop a robotic manipulator mediated system that young children with limited motor function can use to engage into free play, and to measure to what extent such a system increases their play opportunities, and how it improves the children's development. 
The research questions of this study are: 1 . Do bi-manual robotic arm manipulators improve the playfulness of young children with limited motor function when used as play mediators? 2 . Are high-precision robotic arm mediated play interactions beneficial for the development of young children with limited motor function?

To answer these questions, we will conduct a crossover trial with typically developing children and children with CP. The following selection criteria have been established for the participants: children between six and twelve years old, with severe motor impairments, corresponding to levels IV and V of the Gross Motor Function Classification System (GMFCS) [12]. The parents, educators and therapists of these children will also be involved throughout the participatory design process.

With the approval of the Ethics Committee for Research Involving Human Beings of the University of the Basque Country (CEISH), we will first do a preliminary test of the developed system with a convenience sample of typically developing children aged six to twelve years old from local schools. After assessing the results, we will make any fine adjustment to the design of the system and the experiments if needed. We will then proceed to test the same system with a convenience sample of children with CP from local special education schools and therapy centers. The reason for first performing the tests with typically developing children is to refine the system and experiment design to further minimize the exposure of unexpected problems to children with $\mathrm{CP}$, who are part of an even more vulnerable population than the typically developing ones.

Robots with high-precision articulated arms have not been extensively used for research around child play, mostly because they are quite expensive and unsafe for sharing spaces with humans. With the increasing emergence of (industrial) "collaborative" robots, it becomes possible for people to safely work in the same space with them. In addition, collaborative robots of small size and low price are appearing in the market, and these may be ideal for the intermediate manipulation that is to be developed in this project.

Therefore, we propose a work bench composed by a two-armed articulated robot, and its virtual counterpart. The robot will be programmed with a set of games, activities and recorded sequences by which small toys and objects can be seamlessly manipulated.

In order to be able to compare the results with previous studies, we will follow the most widely used and standardized metrics and evaluation methods, such as the Test of Playfulness [5]. Additionally, since in the near future we intend to test the applicability of this robot for the evaluation of cognitive development of children with motor disabilities, we have taken into account the protocol defined by Encarnação et al. [18].

We are currently working on the definition of the child-robot interaction system. To this end, we have applied participatory design for the generation of playful activities that are attractive for children and that promote cognitive development. The user interfaces must be accessible and be based on the principles of the Augmentative and Alternative Communication. In addition, the child-robot interface must be compatible with the application of "shared control" and "mixed initiative" techniques.

With respect to the programming of the bi-manual cooperative robot, we are working on the definition of a language for the manipulation of abstract objects that will be cyclically refined until the tests meet the metrics of acceptability, efficiency and functionality initially defined.

Since the ludic activities should be designed by the tutors, we defined sketches in two and three dimensions to be used for the initial tests. These will allow us to program the robot with basic activities that will serve as a guide to the tutors who will later design the games. The final objective is to compile a set of guidelines that describe the ludic, technical, procedural characteristics, etc. on which the games that are designed for this environment should be based.

\section{Conclusions}

Free play is undoubtedly beneficial for children's cognitive development. However, children with motor restrictions (such as those caused by $\mathrm{CP}$ ) may not have sufficient play opportunities. Robots have been shown to be appropriate mediators for play to be used by children in many recent 
studies $[4,11,14]$. However, we argue that the use of higher-precision robotic manipulators to mediate between children and their play objects can provide children with physical disabilities even more opportunities to engage on free play. Therefore, we have proposed a robotic system to explore this research field.

The main results of this work are, with respect to the child-robot interaction: the design of adaptive interfaces (based on user modeling) that allow children with motor impairments to control (in a restricted way) the movements and actions of the robot. Dimensional reduction methods will be applied to allow the handling of a two-armed robot (each with six degrees of freedom), and to facilitate its control through the user interface. In the area of cognitive ergonomics, methods and metrics will be provided to monitor and quantitatively and qualitatively measure the cognitive development of children who use the robot, in comparison with the evolution of children without motor impairments.

Further results include: a selection of toys and games most suited to the physical and cognitive conditions of children with severe motor impairments; a collaborative interface design methodology (focused on the user) to allow the participation of children and their caregivers in the design of the robot control interface; measures of the emotional impact, immersion, degree of control and cognitive evolution among the children involved; a basic programming environment for articulated arms for the manipulation of toys; and an author tool for the design of games mediated by articulated arms.

Funding: This work was partially funded by the Basque Government, Department of Education, Universities and Research under Grant IT980-16, as well as by the Ministry of Economy and Competitiveness of the Spanish Government and the European Regional Development fund-ERFD (PhysComp project, grant TIN2017-85409-P). Xabier Gardeazabal holds a PhD scholarship from the University of the Basque Country (UPV/EHU) (grant PIF16/212).

Conflicts of Interest: The authors declare no conflict of interest.

\section{References}

1. Missiuna, C.; Pollock, N. Play Deprivation in Children With Physical Disabilities: The Role of the Occupational Therapist in Preventing Secondary Disability. Am. J. Occup. Ther. 1991, 45, 882-888, doi:10.5014/ajot.45.10.882.

2. Adams, K.D.; Rincón, A.M.R.; Puyo, L.M.B.; Cruz, J.L.C.; Medina, M.F.G.; Cook, A.M.; Encarnação, P. An exploratory study of children's pretend play when using a switch-controlled assistive robot to manipulate toys. Br. J. Occup. Ther. 2017, 80, 216-224, doi:10.1177/0308022616680363

3. Besio, S.; Amelina, N. 9 Play in Children with Physical Impairment. In Play Development in Children with Disabilties; Besio, S., Bulgarelli, D., Stancheva-Popkostadinova, V., Eds.; Walter de Gruyter GmbH \& Co KG: Boston, MA, USA, 2016; pp. 120-136. doi:10.1515/9783110522143-011.

4. Ríos-Rincón, A.M.; Adams, K.; Magill-Evans, J.; Cook, A. Playfulness in Children with Limited Motor Abilities When Using a Robot. Phys. Occup. Ther. Pediatr. 2016, 36, 232-246, doi:10.3109/01942638.2015.1076559.

5. Harkness, L.; Bundy, A.C. The Test of Playfulness and Children with Physical Disabilities. Occup. Ther. J. Res. 2001, 21, 73-89, doi:10.1177/153944920102100203.

6. Blanche, E.I. 15 - Play in Children with Cerebral Palsy: Doing With-Not Doing To. In Play in Occupational Therapy for Children, 2nd ed.; Parham, L.D., Fazio, L.S., Eds.; Mosby: Saint Louis, MO, USA, 2008; pp. $375-393$. doi:10.1016/B978-032302954-4.10015-7.

7. Pfeifer, L.I.; Pacciulio, A.M.; Santos, C.A.d.; Santos, J.L.d.; Stagnitti, K.E. Pretend play of children with cerebral palsy. Phys. Occup. Ther. Pediatr. 2011, 31, 390-402, doi10.3109/01942638.2011.572149.

8. Blair, E.; Cans, C.; Sellier, E. Epidemiology of the Cerebral Palsies. In Cerebral Palsy: A Multidisciplinary Approach; Panteliadis, C.P., Ed.; Springer International Publishing: Cham, Switzerland, 2018; pp. 19-28. doi:10.1007/978-3-319-67858-0_3.

9. Okimoto, A.M.; Bundy, A.; Hanzlik, J. Playfulness in children with and without disability: Measurement and intervention. Am. J. Occup. Ther. 2000, 54, 73-82. doi:10.5014/ajot.54.1.73. 
10. Chang, H.J.; Chiarello, L.A.; Palisano, R.J.; Orlin, M.N.; Bundy, A.; Gracely, E.J. The determinants of self-determined behaviors of young children with cerebral palsy. Res. Dev. Disabil. 2014, 35, 99-109. doi:10.1016/j.ridd.2013.10.004.

11. Schulmeister, J.; Wiberg, C.; Adams, K.; Harbottle, N.; Cook, A. Robot assisted play for children with disabilities. In Proceedings of the 29th Annual RESNA Conference, Thriving in Challenging Times: The Future of Rehabilitation Engineering and Assistive Technology, Atlanta, GA, USA, 22-26 June 2006. doi:10.7939/R3RJ48T6P.

12. Palisano, R.; Rosenbaum, P.; Bartlett, D.; Livingston, M. Gross Motor Function Classification System-Expanded and Revised. CanChild Centre for Childhood Disability Research, McMaster University; Institute for Applied Health Sciences McMaster University: Hamilton, ON, Canada, 2007; p. b15.

13. Eliasson, A.C.; Krumlinde-Sundholm, L.; Rösblad, B.; Beckung, E.; Arner, M.; Öhrvall, A.M.; Rosenbaum, P. The Manual Ability Classification System (MACS) for children with cerebral palsy: Scale development and evidence of validity and reliability. Dev. Med. Child Neurol. 2006, 48, 549-554, doi:10.1111/j.1469-8749.2006.tb01313.x.

14. Rios Rincon, A.; Sirard, K.; Wainer, A.; Adams, K. LEGO Robot promoting mother-child communication during free play: A pilot study with a child with severe motor impairment. In Proceedings of the Rehabilitation Engineering and Assistive Technology Society of North America (RESNA) Annual Conference, Bellevue, Washington, 22-24 June 2013.

15. Poletz, L.; Encarnação, P.; Adams, K.; Cook, A. Robot skills and cognitive performance of preschool children. Technol. Disabil. 2010, 22, 117-126. doi:10.3233/TAD-2010-0296.

16. Cook, A.M.; Adams, K.; Volden, J.; Harbottle, N.; Harbottle, C. Using Lego robots to estimate cognitive ability in children who have severe physical disabilities. Disabil. Rehabil. Assist. Technol. 2011, 6, 338-346, doi:10.3109/17483107.2010.534231.

17. Encarnação, P.; Piedade, G.; Cook, A.; Adams, K.; Gil, I.; Maya, C.; Azevedo, L.; Londral, A.R.; Rodrigues, S. Virtual robot and virtual environments for cognitive skills assessment. In Everyday Technology for Independence and Care; Gelderblom, G.J., Soede, M., Adriaens, L., Miesenberger, K., Eds.; Assistive Technology Research Series; IOS Press: Clifton, VA, USA, 2011; Volume 29, pp. 508-516. doi:10.3233/978-1-60750-814-4-508.

18. Encarnação, P.; Alvarez, L.; Rios, A.; Maya, C.; Adams, K.; Cook, A. Using virtual robot-mediated play activities to assess cognitive skills. Disabil. Rehabil. Assist. Technol. 2014, 9, 231-241, doi:10.3109/17483107.2013.782577.

19. Encarnação, P.; Leite, T.; Nunes, C.; da Ponte, M.N.; Adams, K.; Cook, A.; Caiado, A.; Pereira, J.; Piedade, G.; Ribeiro, M. Using assistive robots to promote inclusive education. Disabil. Rehabil. Assist. Technol. 2017, 12, 352-372, doi:10.3109/17483107.2016.1167970.

(C) 2019 by the authors. Licensee MDPI, Basel, Switzerland. This article is an open access article distributed under the terms and conditions of the Creative Commons Attribution (CC BY) license (http:// creativecommons.org/licenses/by/4.0/). 